\title{
IMPROVING THE ACCURACY OF ESTIMATED 3D POSITIONS USING MULTI-TEMPORAL ALOS/PRISM TRIPLET IMAGES
}

\author{
J. Susaki*,H. Kishimoto \\ Department of Civil and Earth Resources Engineering, Graduate School of Engineering, Kyoto University; \\ Email: \{susaki.junichi.3r, kishimoto.hotsuma.74r\}@kyoto-u.ac.jp
}

Commission III, WG III/1

KEY WORDS: ALOS/PRISM, DSM, triplet images, RPC model, Multi-temporal images

\begin{abstract}
:
In this paper, we present a method to improve the accuracy of a digital surface model (DSM) by utilizing multi-temporal triplet images. The Advanced Land Observing Satellite (ALOS) / Panchromatic Remote-sensing Instrument for Stereo Mapping (PRISM) measures triplet images in the forward, nadir, and backward view directions, and a DSM is generated from the obtained set of triplet images. To generate a certain period of DSM, multiple DSMs generated from individual triplet images are compared, and outliers are removed. Our proposed method uses a traditional surveying approach to increase observations and solves multiple observation equations from all triplet images via the bias-corrected rational polynomial coefficient (RPC) model. Experimental results from using five sets of PRISM triplet images taken of the area around Saitama, north of Tokyo, Japan, showed that the average planimetric and height errors in the coordinates estimated from multi-temporal triplet images were $3.26 \mathrm{~m}$ and $2.71 \mathrm{~m}$, respectively, and that they were smaller than those generated by using each set of triplet images individually. As a result, we conclude that the proposed method is effective for stably generating accurate DSMs from multi-temporal triplet images.
\end{abstract}

\section{INTRODUCTION}

Digital surface models (DSMs) and digital elevation models (DE $\mathrm{Ms}$ ) are widely used for topographic analyses in various fields. For example, in the field of disaster mitigation and management, simulations of flood, tsunami, lava flow from volcanoes, and landslide often use DSM or DEM. The simulation of flood and tsunami in relatively flat areas, in particular, requires a highly accurate DSM or DEM, with accuracy needed to within the tens of centimeters. A DSM models the heights of objects on the ground, whereas a DEM models the heights of the ground when objects are virtually removed. Elevation data directly measured or generated from data measured by aerial sensors are equivalent for DSM. Some topographic products derived from satellite and spaceborne imagery are called DEM even though they are equivalent to DSM. Throughout this paper, we follow the terminology used in the literature.

Highly accurate DSMs can be constructed by use of light detection and ranging (LiDAR) data, which is accurate to within $\pm 15 \mathrm{~cm}$, and accordingly, a highly accurate DEM is generated by filtering the DSM. Airborne LiDAR is now popular for measurement, but the coverage is much more limited than satellitebased imagery. In contrast, interferometric synthetic aperture radar (InSAR) imagery can be used to generate height data. As an application, global topographic data have been generated from shuttle radar topography mission (SRTM) data. However, these data have a relatively low spatial resolution (approximately $90 \mathrm{~m}$ ) and large geolocation and height errors (Rodríguez et al., 2006). Gorokhovich and Voustianiouk (2006) improved the accuracy of the original SRTM 90-m DEM, but the vertical errors are still not small, at $7.58 \pm 0.60 \mathrm{~m}$ in Phuket, Thailand and $4.07 \pm 0.47$ $m$ in New York, USA (mean \pm standard error of the mean). In addition, the improved DEMs are sensitive to both slope and aspect characteristics of the terrain. Therefore, optical imagery is

* Corresponding author. still the preferred choice for providing high-resolution DSM with high accuracy to cover a wide area.

Various techniques to improve the accuracies of DSMs and DEMs derived from optical sensor data have been investigated: block adjustment calibration (Bouillon et al., 2006), multi-scale water body detection (Fujisada et al., 2011), and dynamic triangle constraint in image matching (Zhu et al., 2007) are of particular note. One recent advance is to use triplet (or greater) imagery. For example, Zhang and Gruen (2006) used more than two IKONOS images simultaneously to generate DSMs by combining the matching results of feature points, grid points, and edges by employing a coarse-to-fine strategy. Giribabu et al. (2013) examined the accuracies of DEMs generated from stereo triplet Cartosat-1 images with a spatial resolution of $2.5 \mathrm{~m}$ at the ground. In that method, the planimetric and height root-meansquare errors (RMSEs) were approximately $2.5 \mathrm{~m}$ and $2.95 \mathrm{~m}$, respectively.

The Advanced Land Observing Satellite (ALOS) Panchromatic Remote-sensing Instrument for Stereo Mapping (PRISM) measures triplet imagery in the forward, nadir, and backward view directions. The obtained triplet images are used to generate DSMs by applying a rational polynomial coefficient (RPC) model (Fraser et al., 2002; Grodecki and Dial, 2003; Fraser et al., 2006). Each image is composed of data measured by several charge-coupled device (CCD) units, and the coefficients of the RPC models are calculated per unit. To generate a global DSM from the data, stacking and mosaicking are applied to multi-temporal scenebased DSMs. During processing, any height bias is detected and removed by referring to a height reference, the Ice, Cloud, and land Elevation Satellite (ICESat) and SRTM version-2 data. The height accuracy of DSMs generated by stacking and mosaicking ranged from about $3.0 \mathrm{~m}$ to $6.0 \mathrm{~m}$ for most test sites, with a target accuracy of $5.0 \mathrm{~m}$ (Tadono et al., 2014). This approach may not be robust to errors because the detection of errors depends on the accuracy of the reference data. In this paper, we take a different approach to improving DSM accuracy: we de- 
termine three-dimensional (3D) coordinates at points of interest by solving observation equations composed from multi-temporal observations.

The remainder of this paper is organized as follows. An explanation of the proposed method is given in Section 2. The target location and data collected from the site are described, and the experimental results are reported, in Section 3. The implications of these results and the validity of the algorithm are then discussed in Section 4. Finally, Section 5 concludes the paper.

\section{METHOD}

\section{$2.1 \quad$ RPC Model}

An RPC model expresses transformations between image coordinates. These are characterized by a line $l$ and sample $s$ along with the latitude $\phi$, longitude $\lambda$, and ellipsoidal height $h$ at a specific point. The data are normalized by using the scales $l_{s}, s_{s}, \phi_{s}, \lambda_{s}, h_{s}$ and offsets $l_{0}, s_{0}, \phi_{0}, \lambda_{0}, h_{0}$; these are applied to the variables $l, s, \phi, \lambda$ and $h$, respectively, which are thereby normalized to $l_{n}, s_{n}, \phi_{n}, \lambda_{n}$, and $h_{n}$

$$
\begin{aligned}
& l=l_{s} l_{n}+l_{0} \\
& s=s_{s} s_{n}+s_{0} \\
& \phi_{n}=\frac{\phi-\phi_{0}}{\phi_{s}} \\
& \lambda_{n}=\frac{\lambda-\lambda_{0}}{\lambda_{s}} \\
& h_{n}=\frac{h-h_{0}}{h_{s}}
\end{aligned}
$$

The RPC model comprises the quotients of two third-order polynomials, expressed as Equation (7).

$$
\begin{aligned}
l_{n} & =\frac{f_{1}\left(\phi_{n}, \lambda_{n}, h_{n}\right)}{f_{2}\left(\phi_{n}, \lambda_{n}, h_{n}\right)} \\
s_{n} & =\frac{f_{3}\left(\phi_{n}, \lambda_{n}, h_{n}\right)}{f_{4}\left(\phi_{n}, \lambda_{n}, h_{n}\right)}
\end{aligned}
$$

Here,

$$
\begin{aligned}
& f_{i}\left(\phi_{n}, \lambda_{n}, h_{n}\right)=a_{i 1}+a_{i 2} \lambda_{n}+a_{i 3} \phi_{n}+a_{i 4} h_{n} \\
+ & a_{i 5} \lambda_{n} \phi_{n}+a_{i 6} \lambda_{n} h_{n}+a_{i 7} \phi_{n} h_{n}+a_{i 8} \lambda_{n}^{2} \\
+ & a_{i 9} \phi_{n}^{2}+a_{i 10} h_{n}^{2}+a_{i 11} \lambda_{n} \phi_{n} h_{n}+a_{i 12} \lambda_{n}^{2} \phi_{n} \\
+ & a_{i 13} \lambda_{n}^{2} h_{n}+a_{i 14} \lambda_{n} \phi_{n}^{2}+a_{i 15} \phi_{n}^{2} h_{n}+a_{i 16} \lambda_{n} h_{n}^{2} \\
+ & a_{i 17} \phi_{n} h_{n}^{2}+a_{i 18} \lambda_{n}^{3}+a_{i 19} \phi_{n}^{3}+a_{i 20} h_{n}^{3} \lambda_{n},
\end{aligned}
$$

and $a_{i j}(j=1, \cdots, 20)$ denote coefficients.

Equations (1) and (2) are rewritten as Equation (9) by Taylor expansion.

$$
\begin{aligned}
\left(\begin{array}{c}
l \\
s
\end{array}\right)= & \left(\begin{array}{ccc}
\partial l / \partial \phi & \partial l / \partial \lambda & \partial l / \partial h \\
\partial s / \partial \phi & \partial s / \partial \lambda & \partial s / \partial h
\end{array}\right)\left(\begin{array}{c}
\delta \phi \\
\delta \lambda \\
\delta h
\end{array}\right) \\
& +\left(\begin{array}{c}
l^{0} \\
s^{0}
\end{array}\right)
\end{aligned}
$$

Here, $l^{0}$ and $s^{0}$ denote, respectively, the line and sample of the image coordinates corresponding to the approximate object coordinates.

\subsection{Bias-corrected RPC Model}

Now, we introduce a bias-corrected (equivalently, bias-compensated) RPC model by modifying Equations (1) and (2).

$$
\begin{aligned}
l+A_{0} & =l_{s} \frac{f_{1}\left(\phi_{n}, \lambda_{n}, h_{n}\right)}{f_{2}\left(\phi_{n}, \lambda_{n}, h_{n}\right)}+l_{0} \\
s+B_{0} & =s_{s} \frac{f_{3}\left(\phi_{n}, \lambda_{n}, h_{n}\right)}{f_{4}\left(\phi_{n}, \lambda_{n}, h_{n}\right)}+s_{0}
\end{aligned}
$$

Here, $A_{0}$ and $B_{0}$ denote translations of the line and sample coordinates, respectively. These translation parameters can be determined from one or more sets of image coordinates $(l$ and $s)$ and object-space coordinates $(\phi, \lambda$, and $h)$.

\subsection{Residual Equations Using Multi-temporal Triplet Im- ages}

Each set of triplet images has six residual equations. It is assumed that $n$ sets are available. The six residuals are expressed by Equation (12)

$$
\boldsymbol{v}_{\boldsymbol{i}}=\left(\begin{array}{c}
v_{i, F, l} \\
v_{i, F, s} \\
v_{i, N, l} \\
v_{i, N, s} \\
v_{i, B, l} \\
v_{i, B, s}
\end{array}\right)
$$

Here, $i$ indicates the $i$ th set of images, and $F, N$, and $B$ denote the forward, nadir, and backward directions, respectively. The residual equations are derived by using Equation (12).

$$
\boldsymbol{v}_{\text {all }}=\boldsymbol{G}_{\text {all }} \boldsymbol{\Delta}+\boldsymbol{d}_{\text {all }} ; \quad \boldsymbol{P}
$$

Here, $\boldsymbol{P}$ denotes the image measurement precision, and

$$
\boldsymbol{v}_{\text {all }}=\left(\begin{array}{c}
\boldsymbol{v}_{\mathbf{1}} \\
\boldsymbol{v}_{\mathbf{2}} \\
\vdots \\
\boldsymbol{v}_{\boldsymbol{n}}
\end{array}\right), \quad \boldsymbol{\Delta}=\left(\begin{array}{c}
\delta \phi \\
\delta \lambda \\
\delta h
\end{array}\right)
$$

$$
\boldsymbol{G}_{\text {all }}=\left(\begin{array}{ccc}
\partial l_{1, F} / \partial \phi & \partial l_{1, F} / \partial \lambda & \partial l_{1, F} / \partial h \\
\partial s_{1, F} / \partial \phi & \partial s_{1, F} / \partial \lambda & \partial s_{1, F} / \partial h \\
\partial l_{1, N} / \partial \phi & \partial l_{1, N} / \partial \lambda & \partial l_{1, N} / \partial h \\
\partial s_{1, N} / \partial \phi & \partial s_{1, N} / \partial \lambda & \partial s_{1, N} / \partial h \\
\partial l_{1, B} / \partial \phi & \partial l_{1, B} / \partial \lambda & \partial l_{1, B} / \partial h \\
\partial s_{1, B} / \partial \phi & \partial s_{1, B} / \partial \lambda & \partial s_{1, B} / \partial h \\
\vdots & \vdots & \vdots \\
\partial l_{n, F} / \partial \phi & \partial l_{n, F} / \partial \lambda & \partial l_{n, F} / \partial h \\
\partial s_{n, F} / \partial \phi & \partial s_{n, F} / \partial \lambda & \partial s_{n, F} / \partial h \\
\partial l_{n, N} / \partial \phi & \partial l_{n, N} / \partial \lambda & \partial l_{n, N} / \partial h \\
\partial s_{n, N} / \partial \phi & \partial s_{n, N} / \partial \lambda & \partial s_{n, N} / \partial h \\
\partial l_{n, B} / \partial \phi & \partial l_{n, B} / \partial \lambda & \partial l_{n, B} / \partial h \\
\partial s_{n, B} / \partial \phi & \partial s_{n, B} / \partial \lambda & \partial s_{n, B} / \partial h
\end{array}\right)
$$

$$
\boldsymbol{d}_{\text {all }}=\left(\begin{array}{c}
l_{1, F}^{0}-l_{1, F}-A_{1, F} \\
s_{1, F}^{0}-s_{1, F}-B_{1, F} \\
l_{1, N}^{0}-l_{1, N}-A_{1, N} \\
s_{1, N}^{0}-s_{1, N}-B_{1, N} \\
l_{1, B}^{0}-l_{1, B}-A_{1, B} \\
s_{1, B}^{0}-s_{1, B}-B_{1, B} \\
\vdots \\
l_{n, F}^{0}-l_{n, F}-A_{n, F} \\
s_{n, F}^{0}-s_{n, F}-B_{n, F} \\
l_{n, N}^{0}-l_{n, N}-A_{n, N} \\
s_{n, N}^{0}-s_{n, N}-B_{n, N} \\
l_{n, B}^{0}-l_{n, B}-A_{n, B} \\
s_{n, B}^{0}-s_{n, B}-B_{n, B}
\end{array}\right),
$$


with $A_{i, j}$ and $B_{i, j}(i=1, \cdots, n, j=F, N, B)$ as translations applied to the line and samples in the $i$ th set of image $j$.

Then, the function of the sum of squared residuals is defined as

$$
\min \boldsymbol{v}_{\text {all }}^{T} \boldsymbol{P} \boldsymbol{v}_{\text {all }} \text {. }
$$

Finally, $\Delta$ is derived by solving Equation (17).

$$
\boldsymbol{\Delta}=\left(\boldsymbol{G}_{\text {all }}^{T} \boldsymbol{P} \boldsymbol{G}_{\text {all }}\right)^{-1} \boldsymbol{G}_{\text {all }}^{T} \boldsymbol{P} \boldsymbol{d}_{\text {all }}
$$

\section{EXPERIMENTS}

\subsection{Study Areas and Data Used}

We selected Saitama, north of Tokyo, Japan, because five sets of ALOS/PRISM triplet images (Level 1B1) were available. The acquisition dates are 31 Oct $2006\left(T_{1}\right), 31 \mathrm{Jan} 2007\left(T_{2}\right), 3$ May $2007\left(T_{3}\right), 24 \operatorname{Dec} 2009\left(T_{4}\right)$, and 27 Dec $2010\left(T_{5}\right)$. Each image is composed of four subimages acquired by different CCD units. The CCD-unit-based RPC and 39 ground points were provided by the Japan Aerospace Exploration Agency (JAXA). From among the 39 ground points, 4 were used as ground control points to determine $A_{i, j}$ and $B_{i, j}(i=1, \cdots, n, j=F, N, B)$. The other 35 points were used as checkpoints (CKPs) to assess geolocational errors.

\subsection{Results}

We estimated the object coordinates of 35 CKPs from image coordinates in two different ways: using individual triplet images separately, and using five triplet images simultaneously. The RMSEs for planimetric position, height, and 3D position were calculated. Figure 1 shows the planimetric and height errors of CKPs on images, obtained by applying the standard RPC model without bias correction. Figure 2 shows the corresponding results with bias correction. Figure 3 shows the planimetric, height and 3D RMSEs of the two RPC models. Tables 1 and 2 show the RMSEs and maximum errors of the results obtained by using the standard RPC model and the bias-corrected RPC model, respectively.

\section{DISCUSSION}

\subsection{Estimates Using Multi-temporal Triplet Images}

Figure 3 and Tables 1 and 2 show that the accuracies of the results obtained by using multi-temporal triplet images are better than those obtained by using triplet images individually in most cases. The theory of the least-squares method (LSM) indicates that increasing the number of observations will result in more precise estimates so long as systematic errors are removed and only unbiased random errors are left. The results shown in Figure 3 and Tables 1 and 2 suggest that LSM is appropriate for this application, and Figures 1 and 2 demonstrate that large errors may be excluded by solving through LSM. In addition, the maximum planimetric, height, and 3D errors in Tables 1 and 2 were much reduced by the use of multi-temporal triplet images.

We also examined the dependency of geolocational errors on topography. According to Figures 1 and 2, planimetric and height errors in hilly areas are not notably larger than those in flat areas. When we applied the standard and bias-corrected RPC models to another area, Osaka, in the western part of Japan, the results were also independent of topography. These results support the conclusion that the RPC model is robust to topographic effects.

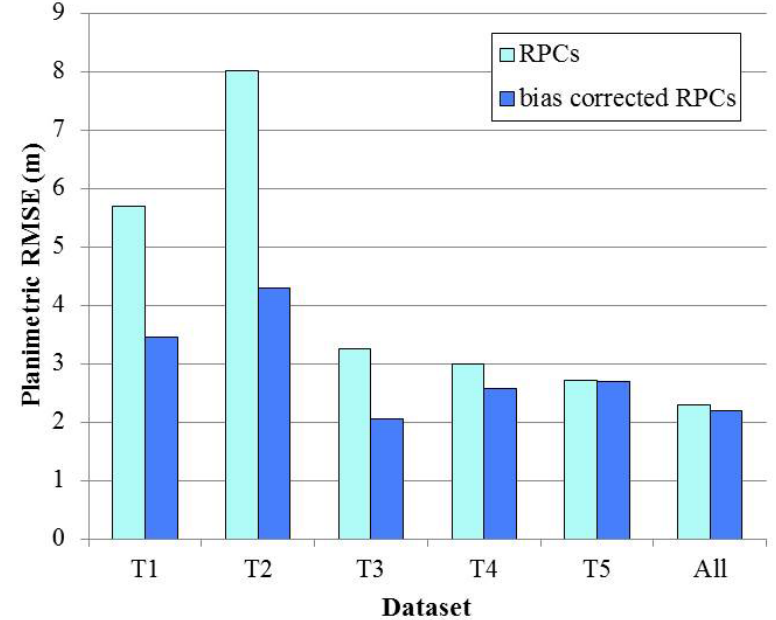

Planimetric

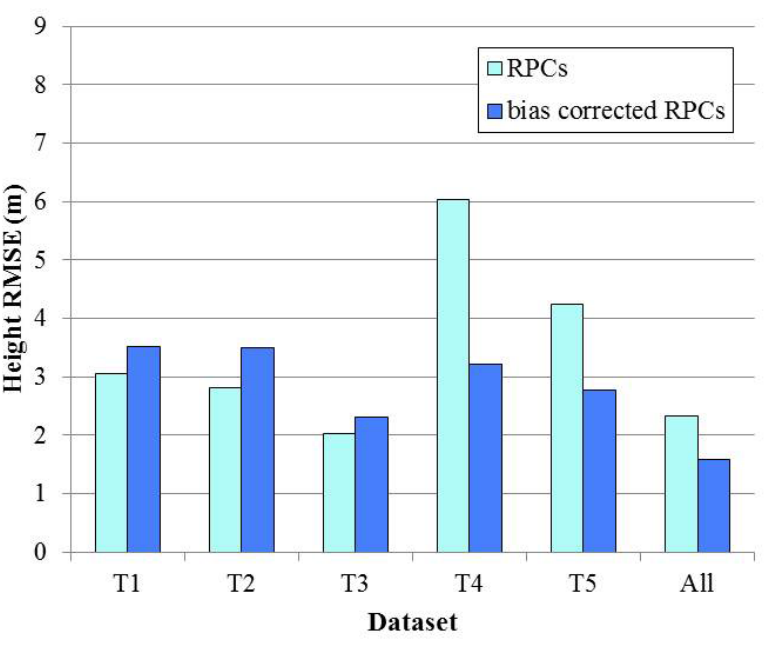

Height

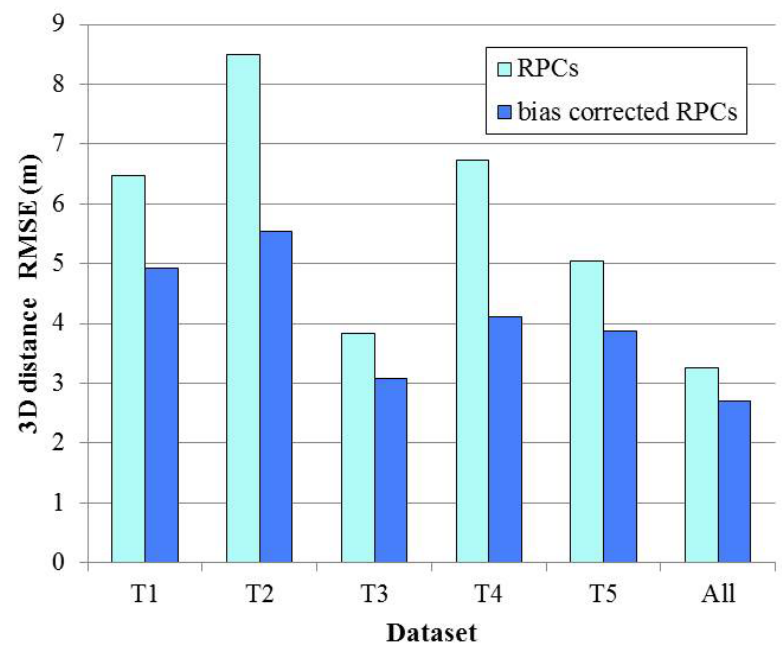

$3 \mathrm{D}$

Figure 3. RMSE of coordinates estimated by using the standard RPC model and the bias-corrected RPC model 


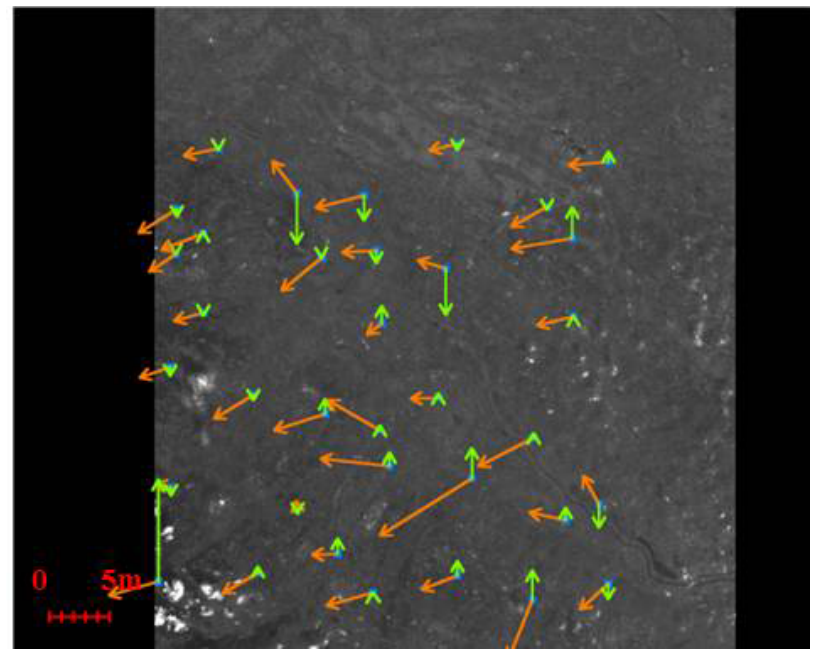

$T_{1}$

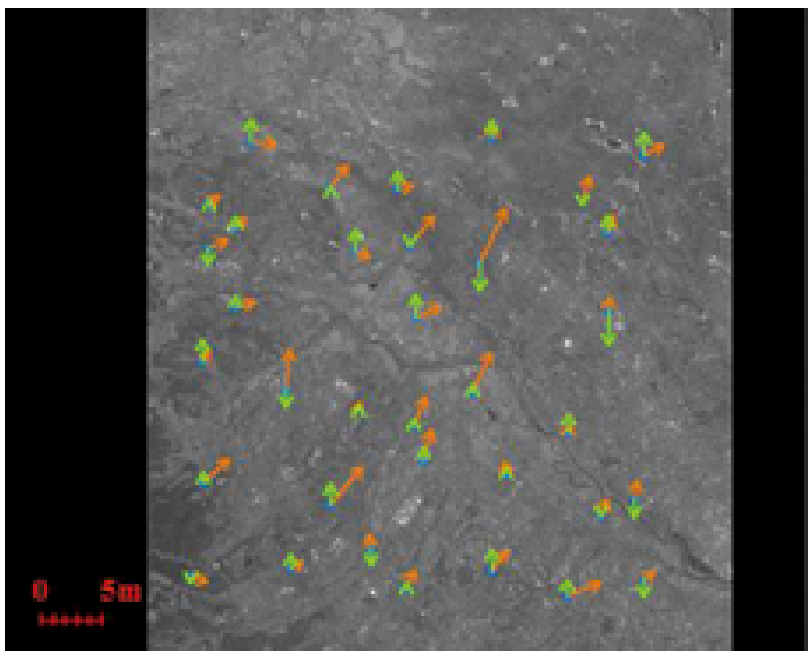

$T_{3}$

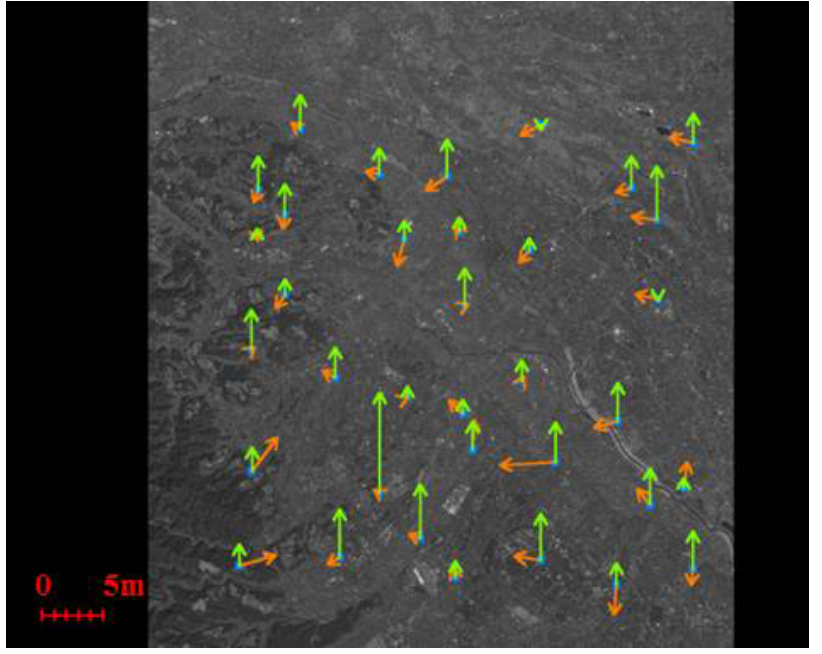

$T_{5}$

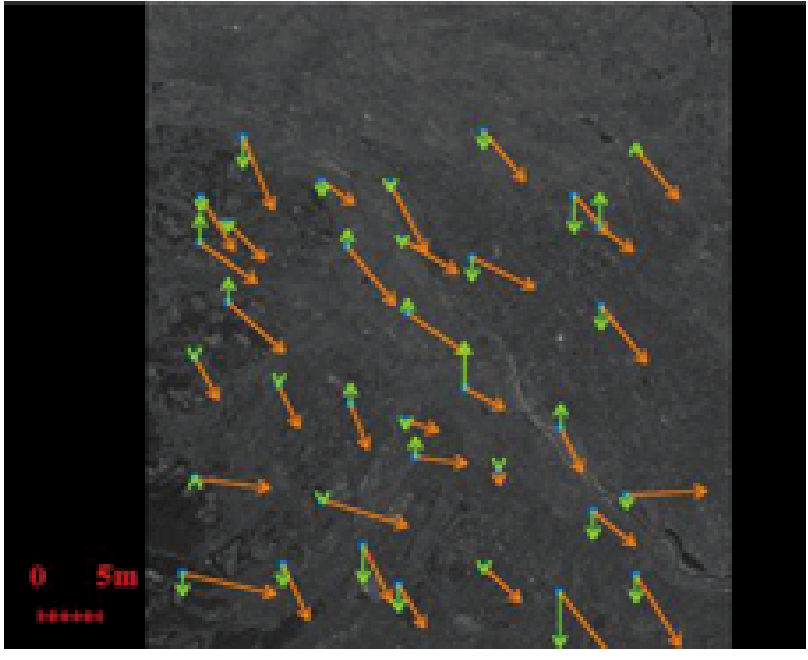

$T_{2}$

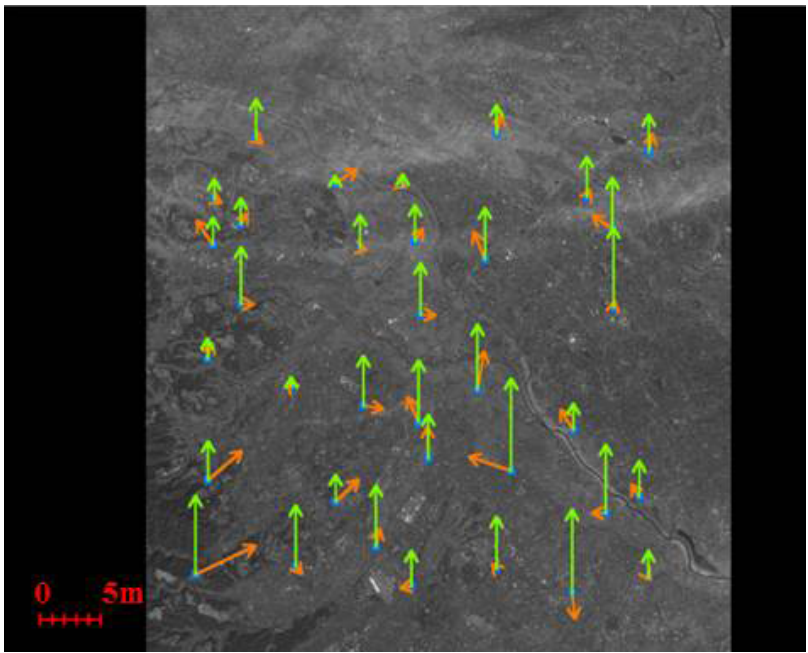

$T_{4}$

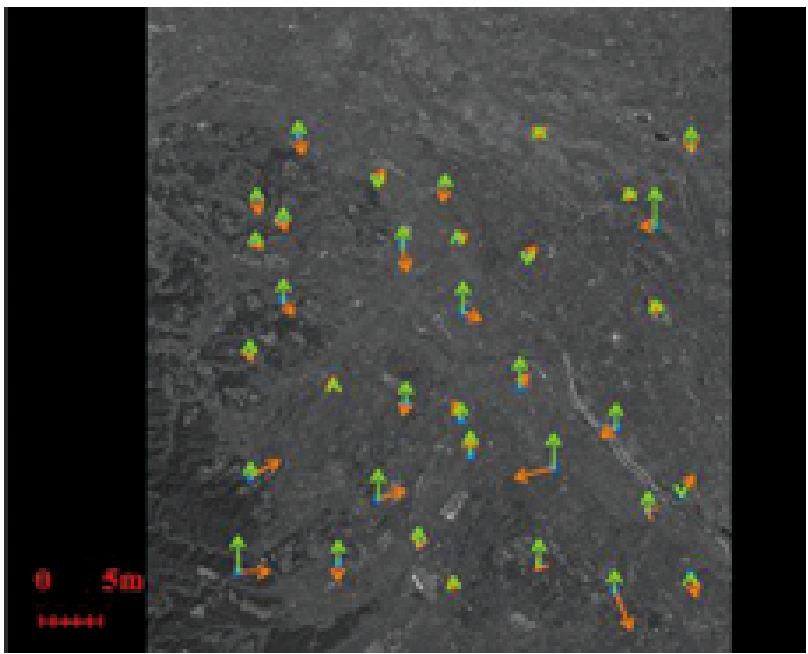

All

Figure 1. RMSE of coordinates estimated by using the standard RPC model without bias correction. Brown and green arrows denote planimetric and height errors, respectively. 


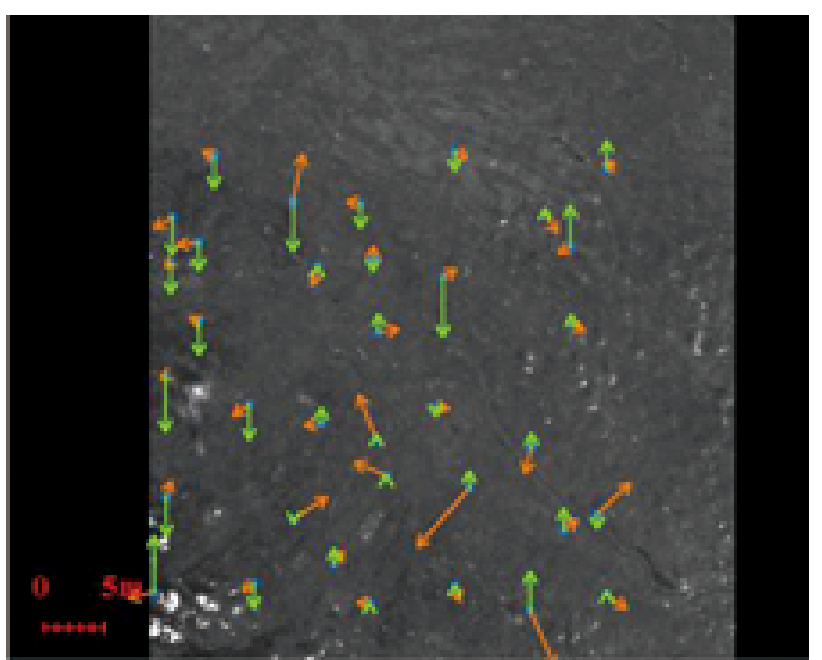

$T_{1}$

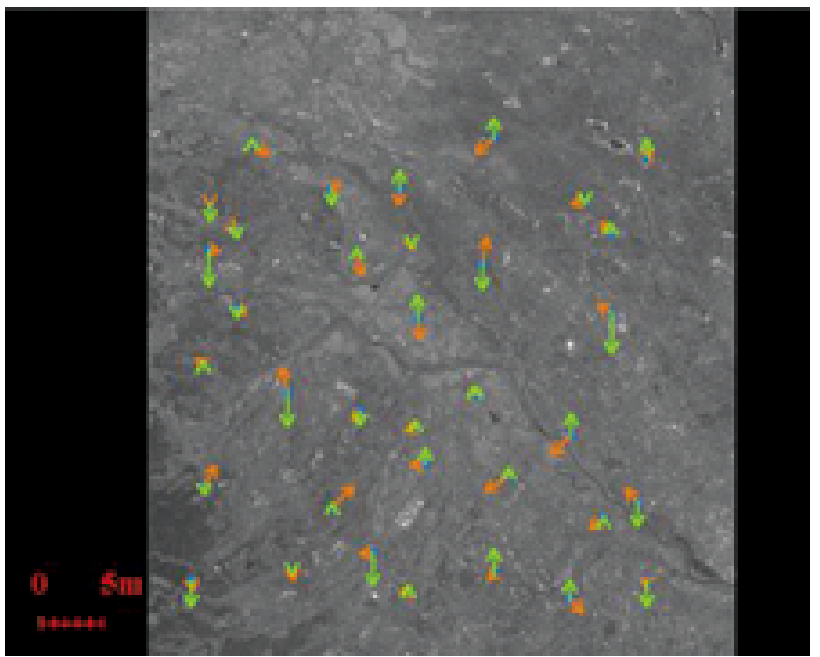

$T_{3}$

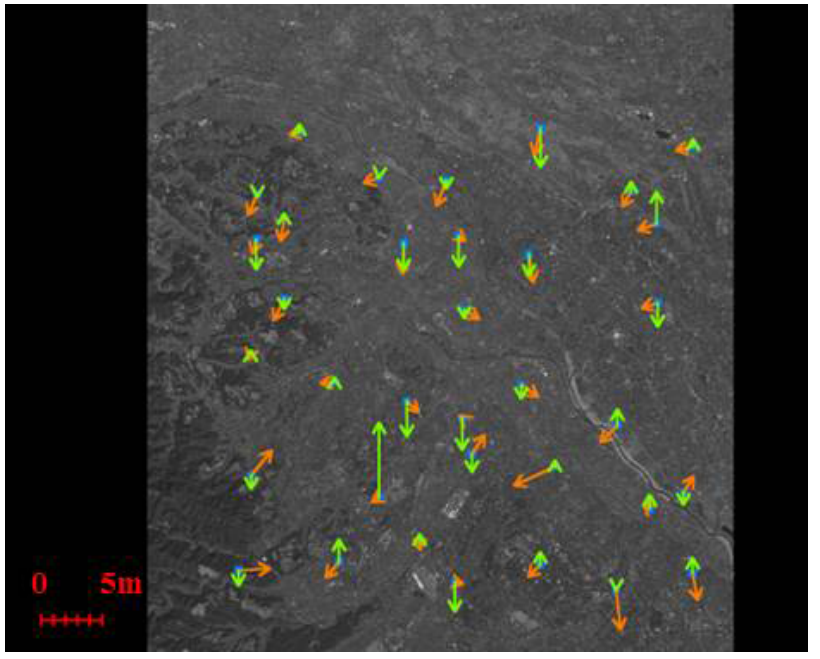

$T_{5}$

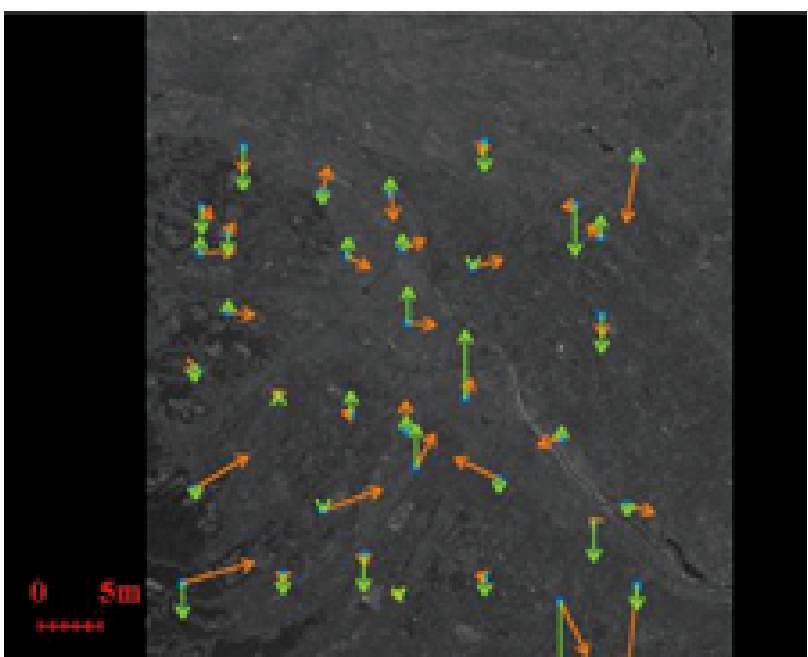

$T_{2}$

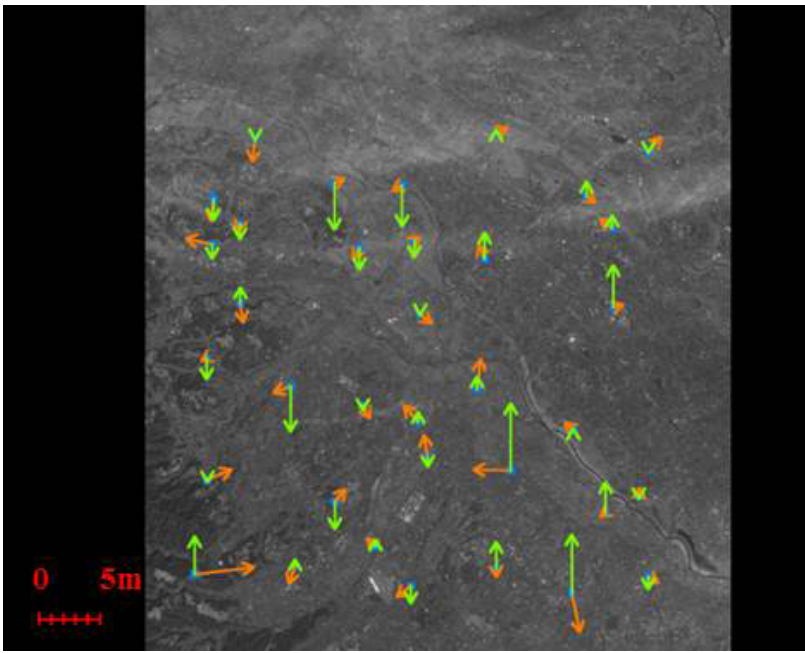

$T_{4}$

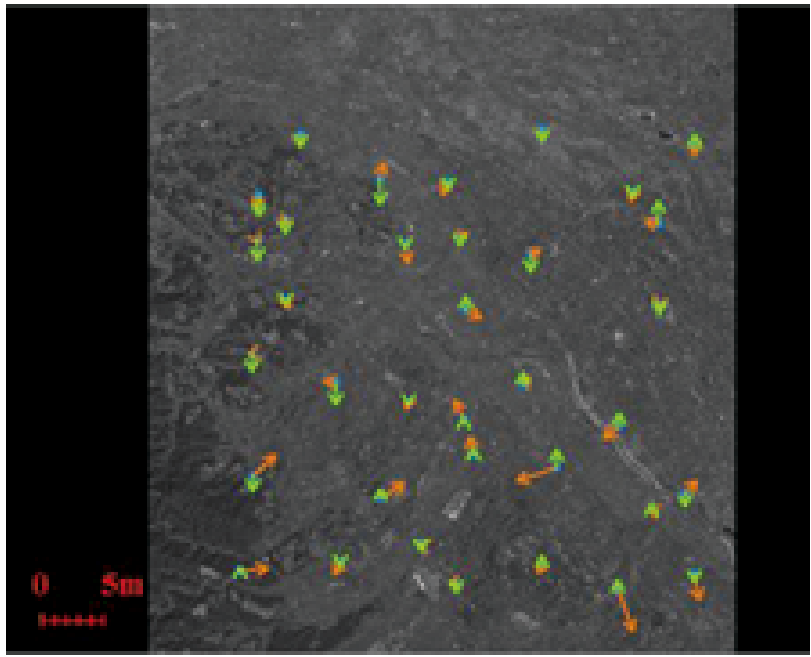

All

Figure 2. RMSE of coordinates estimated by using the bias-corrected RPC model. Brown and green arrows denote planimetric and height errors, respectively. 


\begin{tabular}{|c|c|rrr|rrr|}
\hline Dataset & Number of & \multicolumn{3}{|c|}{ RMSE $(\mathrm{m})$} & \multicolumn{3}{|c|}{ Max error $(\mathrm{m})$} \\
\cline { 3 - 8 } & images & Planimetric & Height & 3D & Planimetric & Height & 3D \\
\hline$T_{1}$ & 3 & 5.70 & 3.06 & 6.47 & 13.51 & 12.68 & 14.21 \\
$T_{2}$ & 3 & 8.02 & 2.82 & 8.50 & 14.19 & 6.81 & 15.74 \\
$T_{3}$ & 3 & 3.26 & 2.02 & 3.83 & 7.40 & 4.21 & 8.05 \\
$T_{4}$ & 3 & 2.99 & 6.02 & 6.73 & 8.27 & 11.43 & 12.79 \\
$T_{5}$ & 3 & 2.72 & 4.24 & 5.04 & 6.80 & 12.41 & 12.44 \\
\hline All & 15 & 2.29 & 2.32 & 3.26 & 5.14 & 4.60 & 6.42 \\
\hline
\end{tabular}

Table 1. Accuracy of object coordinates estimated by using the standard RPC model without bias correction

\begin{tabular}{|c|c|rrr|rrr|}
\hline Dataset & Number of & \multicolumn{3}{|c|}{ RMSE $(\mathrm{m})$} & \multicolumn{4}{|c|}{ Max error $(\mathrm{m})$} \\
\cline { 3 - 8 } & images & Planimetric & Height & 3D & Planimetric & Height & 3D \\
\hline$T_{1}$ & 3 & 3.46 & 3.51 & 4.93 & 9.66 & 7.38 & 9.88 \\
$T_{2}$ & 3 & 4.29 & 3.49 & 5.53 & 10.41 & 8.37 & 11.16 \\
$T_{3}$ & 3 & 2.04 & 2.30 & 3.08 & 3.72 & 4.95 & 5.32 \\
$T_{4}$ & 3 & 2.58 & 3.21 & 4.12 & 7.37 & 8.27 & 9.45 \\
$T_{5}$ & 3 & 2.70 & 2.78 & 3.87 & 5.55 & 9.22 & 9.28 \\
\hline All & 15 & 2.19 & 1.59 & 2.71 & 5.36 & 3.14 & 5.58 \\
\hline
\end{tabular}

Table 2. Accuracy of object coordinates estimated by using the bias-corrected RPC model

\begin{tabular}{|c|rrr|rrr|}
\hline \multirow{2}{*}{ Dataset } & \multicolumn{3}{|c|}{ RMSE (m) } & \multicolumn{3}{c|}{ Max error (m) } \\
\cline { 2 - 7 } & Planimetric & Height & 3D & Planimetric & Height & 3D \\
\hline All & 2.16 & 1.58 & 2.67 & 5.36 & 3.14 & 5.58 \\
\hline
\end{tabular}

Table 3. Accuracy of object coordinates estimated by using the bias-corrected RPC model followed by removing outliers and recalculating

\subsection{Effect and Detection of Land Deformation}

The proposed method uses multi-temporal triplet images acquired during a certain period to estimate object coordinates. The estimated coordinates are assumed to be fixed throughout the period. This indicates that the proposed method implicitly assumes that no land deformation has occurred during the period. In most cases, this assumption is likely to be acceptable. However, suppose that we analyze data from an area where land deformation has occurred. The proposed method estimates the fixed object coordinates of a point of interest. When we examine the planimetric and height residuals, the directions of the residuals may be significantly different from before to after deformation. We expect that such analysis of residuals can be used to discriminate whether land deformation has taken place during the period of data acquisition. In the future, we intend to report the results of such an approach by applying the method to areas where land deformation occurred.

\subsection{Bias Correction}

Figures 1 and 2 and Tables 1 and 2 show the significant difference in errors between the standard RPC model and the bias-corrected RPC model. As a reference, scattergrams of errors of coordinates estimated by using the standard RPC model without bias correction are shown in Figure 4. In general, the results using the standard RPC model show systematic errors, that is, the directions of the errors are similar. However, in this experiment, no significantly clear direction was found. Figure 4 shows that $T_{2}$ in all directions (forward, nadir, and backward) and $T_{5}$ in the backward direction have large systematic errors. Even when systematic errors are observed, the bias-corrected RPC model contributed to improving the accuracies of the estimated object coordinates.
Instead of Equations (1) and (2), Fraser et al. (2006) proposed a practical bias-compensation approach based on affine transformation. The results of applying affine bias correction to the images used in this research were almost same as those obtained by using Equations (1) and (2). Therefore, we selected the simpler transformation expressed by Equations (1) and (2) in this research. However, the similarity of results may not always hold; they could, for example, be dependent on the sensor and its calibration.

\subsection{Outlier Removal}

We extended the proposed method to remove outliers from the observations. First, the bias-corrected RPC model was applied and the residuals between observed image coordinates and estimated image coordinates were calculated. The planimetric image residuals were thereby obtained. Then, outliers with planimetric residuals larger than a designated threshold were removed. Finally, the bias-corrected RPC model was applied to the unremoved observations. Table 3 shows the results obtained by using this approach with a 5-pixel threshold. The results were almost the same as those in Table 2. In general, such approaches to recalculate after removing outliers are effective at improving accuracy. In the future, we intend to examine approaches for achieving even higher accuracy.

\section{CONCLUSIONS}

In this paper, we presented a method to improve the accuracy of a DSM by utilizing multi-temporal triplet images. The approach to estimation of object coordinates of each ground point is based on a bias-corrected RPC model. Assuming that no land deformation occurred during the period of data acquisition, the proposed 


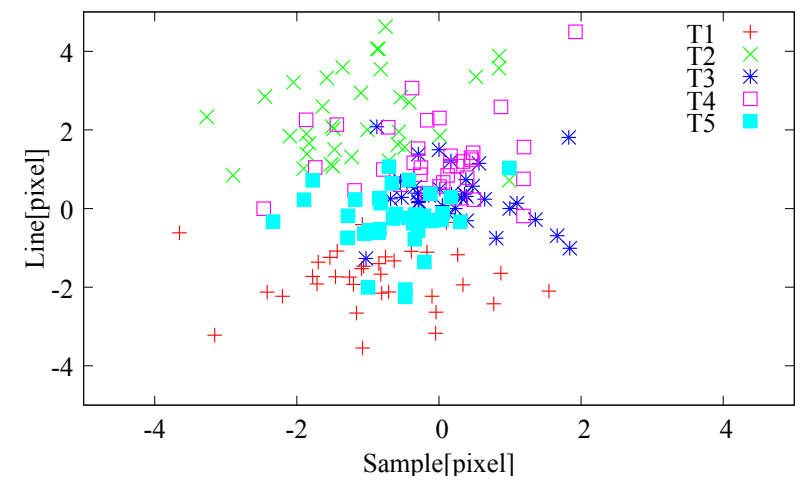

Forward
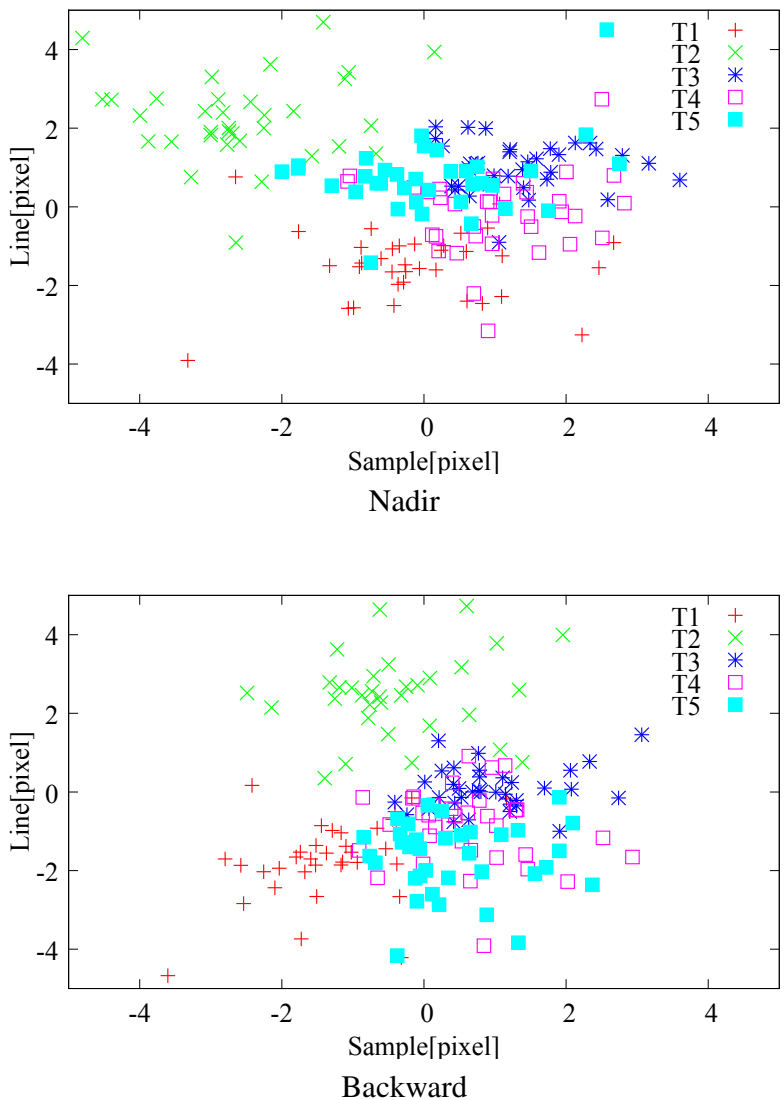

Figure 4. Scattergram of errors of image coordinates estimated by using the standard RPC model without bias correction method can generate estimates by LSM. Experimental results for five sets of PRISM triplet images showed that the planimetric and height errors of the coordinates estimated by using multitemporal triplet images were $3.26 \mathrm{~m}$ and $2.71 \mathrm{~m}$, respectively, and that they were smaller than those generated by using each set of triplet images alone. These results are better than those achieved by stacking and mosaicking multi-temporal DSMs (Tadono et al., 2014). In the future, we intend to apply the proposed method to other areas and to generate PRISM-driven DSMs that are more accurate than the existing DSMs.

\section{ACKNOWLEDGEMENTS}

This research was supported by a Grant-in-Aid for Scientific Research (KAKENHI) for Young Scientists (B) (No. 22760393) and by a program of the Fourth Advanced Land Observing Satellite-2 Research Announcement, Japanese Aerospace Exploration Agency. The authors express thanks to Dr. Takeo Tadono of JAXA for valuable advice about assessing the results obtained in this research.

\section{REFERENCES}

Bouillon, A., Bernard, M., Gigord, P., Orsoni, A., Rudowski, V. and Baudoin, A., 2006. SPOT 5 HRS geometric performance: Using block adjustment as a key issue to improve quality of DEM generation. ISPRS J. Photogram. Remote Sens., pp. 60, 134 146.

Giribabu, D., Rao, S.S. and Murthy, Y.V.N.K., 2013. Improving Cartosat-1 DEM accuracy using synthetic stereo pair and triplet. ISPRS J. Photogram. Remote Sens., 77, pp. 31 - 43.

Gorokhovich, Y. and Voustianiouk, A., 2006. Accuracy assessment of the processed SRTM-based elevation data by CGIAR using field data from USA and Thailand and its relation to the terrain characteristics. Remote Sens. Enviorn., 104, pp. 409 415 .

Grodecki, J. and Dial, G., 2003. Block adjustment of high-resolution satellite images described by rational polynomials. Photogram. Eng. \& Remote Sens., 69, pp. 59 - 68.

Fraser, C.S., Baltsavias, E., and Gruen, A., 2002. Processing of Ikonos imagery for submetre 3D positioning and building extraction. ISPRS J. Photogram. Remote Sens., 56, pp. 177 - 194.

Fraser, C.S., Dial, G., and Grodecki, J., 2006. Sensor orientation via RPCs. ISPRS J. Photogram. Remote Sens., 60, pp. 182 - 194.

Fujisada, H., Urai, M. and Iwasaki, A., 2011. Advanved methodology for ASTER DEM generation. IEEE Trans. Geosci. Remote Sens., 49, pp. 5080 - 5091.

Rodríguez, E., Morris, C.S. and Belz, J.E., 2006. A global assessment of the SRTM performance. Photogram. Eng. \& Remote Sens., 72, pp. 249 - 260.

Schwind, P., Schneider, M., Palubinskas, G., Storch, T., Müller, R. and Richter, R., 2009. Processors for ALOS optical data: Deconvolution, DEM generation, orthorectification, and atmospheric correction. IEEE Trans. Geosci. Remote Sens., 47, pp. 4074 - 4082.

Tadono, T., Ishida, H., Oda, F., Naito, S., Minakawa, K. and Iwamoto, H., 2014. Precise global DEM generation by ALOS 
PRISM. ISPRS Annals Photogram. Remote Sens. Spatial Info. Sci., II-4.

Takaku, J. and Tadono, T., 2009. PRISM on-orbit geometric calibration and DSM performance. IEEE Trans. Geosci. Remote Sens., 47, pp. 4060 - 4073.

Zhang, L. and Gruen, A., 2006. Multi-image matching for DSM generation from IKONOS imagery. ISPRS J. Photogram. Remote Sens., 60, pp. 182 - 194.

Zhu, Q., Wu, B. and Tian, Y., 2007. Propagation strategies for stereo image matching based on the dynamic triangle constraint. ISPRS J. Photogram. Remote Sens., 62, pp. 295 - 308. 